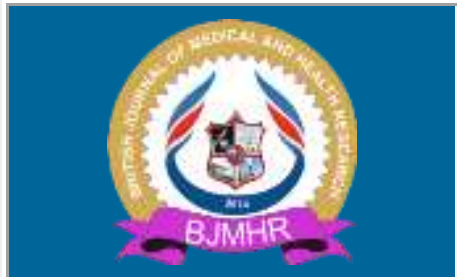

\title{
BJMHR
}

British Journal of Medical and Health Research Journal home page: www.bjmhr.com

\section{Hypersensitivity (Angioedema) Reaction to Omeprazole: A Case Report}

\author{
S.M. Biradar ${ }^{*}$, P. Keerthi Sai ${ }^{1}$, H. Prudhvi Raju ${ }^{1}$, A. Harish kumar ${ }^{1}$, M S. \\ Mulimani $^{2}$, Siddanagouda Biradar ${ }^{2}$, Shivakumar B. ${ }^{1}$. N V. Kalyane ${ }^{1}$ \\ 1.Department of Clinical Pharmacy Practice, SSM College of Pharmacy and Research \\ Centre, Vijaypur-586103. \\ 2.Department of Medicine, Shri B M. Patil Medical College Hospital and Research Centre, \\ Vijaypur-586103.
}

\begin{abstract}
Omeprazole is a substituted benzimidazole that has gained widespread use in the treatment of acidic and peptic ulcer diseases. Though the adverse events with the drug are rare and involve mainly skin inflammation, urticaria, pruritus, alopecia and dry skin have been reported in $0.5-$ $1.5 \%$ of patients. There are very few reports linked to angioedema and urticaria pertaining to Omeprazole or class of PPI as an adverse reaction. Hereby a case of 26-year-old male with hypersensitivity reaction characterized by angioedema (Swelling of lips and fore head). The patient develops adverse drug reaction to Omeprazole may become a hypersensitivity to whole class of PPIs; hence it is to be safer to consider the change of class of drugs for his future correspondence. Simultaneously, all the prescribers should be aware of this possible phenomenon of angioedema while prescribing omeprazole for different clinical indications.
\end{abstract}

Keywords: Adverse drug reactions; Omeprazole ; Angioedema. 


\section{INTRODUCTION}

Proton pump inhibitors (PPIs) are widely being used around the world and it is the secondmost largely sold group of medication. These medications are very potent suppressors of gastric acid secretion, and are highly effective for the management of acid-related gastrointestinal disorders. Since the introduction of Omeprazole, a proton-pump inhibitor (PPI), in 1988, several other PPIs i.e., Lansoprazole, Rabeprazole, Pantoprazole, and Esomeprazole have been developed. ${ }^{1}$ By acting specifically on the proton pump; omeprazole blocks the final step in acid production, thus reducing gastric acidity. They are the most effective drugs used in antiulcer therapy. PPIs are generally safe and well tolerated, with minimum side effects at the rate of $3 \%$ approximately. ${ }^{2}$ The most common side effects include headaches, dizziness, diarrhoea, constipation and cutaneous reactions. ${ }^{2}$ At a lesser frequency, PPI use can result in hepatic dysfunction, vertigo, confusion and haematological disorders. ${ }^{2}$ Though the hypersensitivity reactions are rare with the Omeprazole, several anaphylactic reactions have been reported, some of which describe the presence of cross-reactivity between the different members of the group, although there is no definite pattern has emerged. ${ }^{3}$ Type I hypersensitivity reactions such as urticaria, angioedema, and anaphylaxis to Omeprazole, Lansoprazole, Pantoprazole, and Rabeprazole have been reported. ${ }^{4}$ Around 22 cases of PPI-induced anaphylaxis have been reported in English literature. The onset of symptoms ranged from minutes to hours. The largest case series reported nine patients with omeprazole allergy: urticaria/angioedema in seven and anaphylaxis in two patients. Eight of the patients (88.9\%) showed positive responses to omeprazole with skin prick tests or intradermal tests, which suggested IgE-mediated hypersensitivity. A high level of clinical suspicion and temporal relationship is critical in the diagnosis of PPI-induced hypersensitivity reactions. Skin tests (prick/intradermal test) and controlled challenge tests are helpful in the diagnosis of type I hypersensitivity reactions by PPI. Recently it has been proposed that basophil activation test (BAT) could be helpful in the diagnosis of IgE mediated allergic reactions to PPI as an in-vitro study. ${ }^{4}$

\section{Case:}

A 26-year-old male patient was admitted to a dermatology ward with chief complaints of swelling of lips and fore-head (Angioedema), upon patient medication history interview it has come to know that he had been prescribed Omepazole as one of medication among treatment regimen plan for joint pain. Immediately after taking first dose of omeprazole suddenly he developed swelling over lips and fore-head and gradually progressed in size.

Upon admission treatment was started immediately with Inj. Adrenaline 24 units s/c stat, Inj. Acucort (Hydrocortisone sodium succinate) 100 mg iv-3 days, Tab. Rantac (Ranitidine) 150 mg (1-0-1) half-an-hour before food, Inj. Avil (Pheniramine maleate) 2cc iv stat, Tab. Atarax 
(hydroxyzine hydrochloride) $25 \mathrm{mg}$ (0-0-1). On second day of admission Tab. Aziwok (Azithromycin) $500 \mathrm{mg}$ (1-0-0) for 3 days was prescribed. After four days of treatment the patient general complaints were fair and Patient didn't get any adverse effects associated to $\mathrm{H}_{2}$ receptor (Ranitidine) antagonist.

\section{DISCUSSION:}

Angioedema is a life threatening allergic reaction ${ }^{5}$ which is an area of swelling of deep dermis, subcutaneous, or sub mucosal tissue due to vascular leakage. Acute episodes often involve the lip, eyes, and face; however, angioedema may also affect other parts of body, including respiratory and gastrointestinal mucosa. ${ }^{6}$ Even though angioedema and urticaria are the rate adverse effects to omeprazole, still there are few reports available in the literature. The adverse reaction to omeprazole was induced by an IgE-mediated hypersensitivity mechanism to omeprazole itself and not to a metabolite. ${ }^{7}$

As PPIs are generally well-tolerated, PPI-induced hypersensitivity reactions can occur. These include urticaria, angioedema, anaphylaxis, cytopenia, vasculitis, acute allergic interstitial nephritis, contact dermatitis, drug rash with eosinophilia and systemic symptoms, StevensJohnson syndrome, and toxic epidermal necrolysis. Patients with PPI-related hypersensitivity fits into one of three categories: (1) hypersensitive to all PPIs; (2) Selectively hypersensitive to PPIs, or (3) hypersensitive to one PPI and tolerant of all others. ${ }^{8}$ In the present case report it is not studied adverse drug reaction to any other PPI except Omeprazole, as all PPI potential enough to cause the hypersensitivity reactions including angioedema. ${ }^{9,10,11 .}$

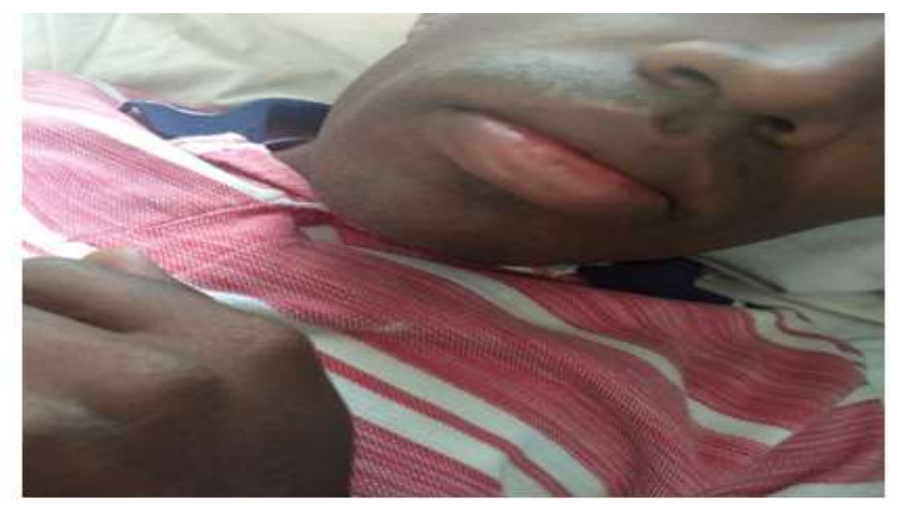

Figure 1: Angioedema (Swelling of Lips)

\section{CONCLUSION:}

Hypersensitivity reactions to PPI may be immediate or nonimmediate reactions, and vary from mild to severe and sometimes even it is life-threatening. A high level of clinical suspicion is critical in the diagnosis of PPI-induced hypersensitivity reactions. Physicians should be aware of the possibility of hypersensitivity reactions to PPI, the mechanisms of which remain largely unclear. A patient may tolerate an alternative PPI or may be allergic to the entire class of drugs. ${ }^{9}$ In the current case report patient hypersensitivity to omeprazole and it is expected to develop 
the same reactions with the whole class of drugs, as supported by the literature, ${ }^{9,10}$ hence it is suggested and practiced in the present case report by changing the class of drug i.e. Ranitidine (higher doses of $\mathrm{H}_{2}$ blockers) alternative to $\mathrm{PPI}^{10}$ for his future correspondence and did not developed any of adverse drug reactions.

\section{REFERENCES:}

1. Sangey C L, Ram Naresh A, Caroline K, et.al. Anaphylactic reaction to omeprazole. Trop gastroenterol 2019: 1-2.

2. Prasad T, Naga kishore Ch, Kalaiselvan V, Gyanendra N S. Omeprazole induced skin hyperpigmentation. Open access journals 2019:1-2.

3. Lobera T, Navarro B, Del pozo M D, Gonzalez I, et.al. Nine cases of omeprazole allergy: cross-reactivity between proton pump inhibitors. $\mathbf{J}$ investing Allergol clin Immunol 2009; 19(1):57-60.

4. Yoon-seok chang. Hypersensitivity reactions to proton pump inhibitors. Curr opin allergy clin immunol 2012; 12(4):348-353.

5. Bowlby H A, Dickens G R. Angioedema and urticaria associated with omeprazole confirmed by drug rechallenge. Pharmacotherapy 1994; 14(1):119-22.

6. https://emedicine.medscape.com/article/135208-overview.

7. Gallindo P A, Borja J, Feo F, et.al. Anaphylaxis to omeprazole. Ann allergy asthma immunol 1999; 82(1):52-4.

8. Sarah A, Sarah C. Hypersensitivity reaction to omeprazole in a patient treated for helicobacter pylori. Ther adv gastroenterol 2015; 8(2):101-104.

9. Lombardo C, Bonadonna P. Hypersensitivity reactions to proton pump inhibitors. Current treatment options in allergy 2015; 2:110-123.

10. Harleen C. P2108-Proton pump inhibitor-induced angioedema. American college of gastroenterology 2019; 1-3.

11. Alexa P K, Jane E, Bruce L Z, Sandra C C. Recurrent anaphylaxis linked to pantoprazole. J allergy clin immunol 2004; 114(4):1-3.

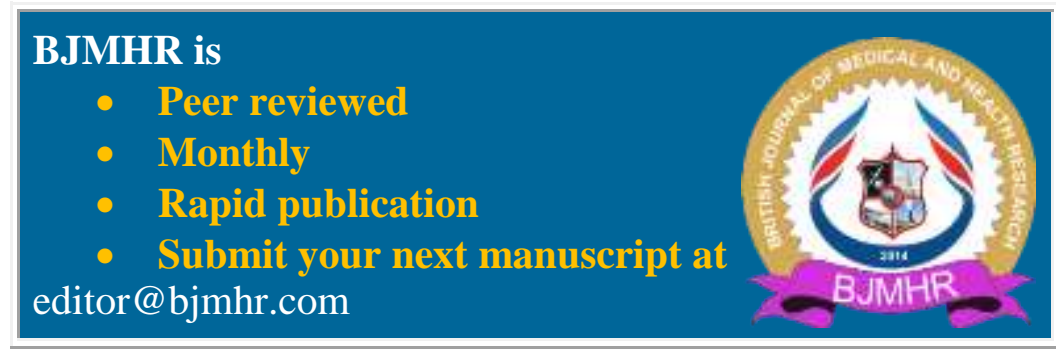

